\title{
Analysis of Circuit Breaker and Relays in Substations
}

\author{
N. Subha Lakshmi and S. Sarumathi
}

\begin{abstract}
There is a necessity of circuit breaker replacement with Gas circuit breaker is to meet the day by day growing load demand, to reduce the down time to upgrade the system with current technology and to provide better protection to the substation power equipment's. To improve the efficiency and reliability and to provide the best quality of power and service to the end customer. Electromechanical relays will lag in functionalities and operations in compression with the current technology numerical relays. Numerical relays are more flexible in configuring the relay settings, having better reliability, multifunctional capability, multiple relay setting characteristics, digital communication capabilities. Numerical relays are modular based so it can be easily serviced/ replaced, Numerical relays have very less burden, having better sensitivity/selectivity, High speed operation (set/ reset), comprises auto resetting and self diagnostics features. Consumes very less space in comparison with electromechanical relays. In this paper the procedure for Gas circuit breaker and numerical relay replacement is illustrated with the substation reference drawing.
\end{abstract}

\section{INTRODUCTION}

$\mathrm{P}$ ROTECTIVE relays play a vital role in the operation of the electrical power system. Protective relays are designed to take action when abnormal conditions occur such as short circuits, overload condition and loss of system synchronism. Protection schemes have typically been made up of discrete components such as over current relays, distance relays, auxiliary relays, and reclosing relays. All of the devices must be wired together to have a complete, functional scheme, which means time and money in the design, development, and installation process. Due to the number of components that make up these protection schemes, detailed installation tests, and routine maintenance programs can be performed to ensure that the schemes are functioning correctly. This requires a significant investment in time, money, and manpower. Increased growth in power systems both in size and complexity has brought about the need for fast, accurate and reliable protective schemes-to protect major equipment and to maintain system stability.

Numerical relays offer many advantages over schemes using discrete components. The overall scheme takes up less

N. Subha Lakshmi, Assistant Professor, Department of EEE, Sri Krishna College of Engineering and Technology, Coimbatore. E-mail: nsubhalakshmi90@gmail.com

S. Sarumathi, Assistant Professor, Department of EEE, Sri Krishna College of Engineering and Technology, Coimbatore. E-mail: sarumathis@skcet.ac.in

DOI:10.9756/BIJPSIC.8355 panel space. The number of components is greatly reduced. The design and wiring is simpler and less costly to implement. Installation and maintenance testing can be greatly reduced. Numerical relay offer many advanced features and functions in addition to their basic protection functions, for instance, fault locating, event reporting, and self-checking. The added benefits of simple scheme design and improved reliability make them a very attractive option. The relay implement more flexible protection schemes, reduce maintenance, and obtain more information to increase understanding of the power system, and improve the reliability of the protection system as a whole at a cost less than conventional electromechanical relays.

\section{GENERAL DESCRIPTIONS}

\section{A. Oil Circuit Breaker}

These breakers employ a high pressure *air-blast as an arc quenching medium. The contacts are opened in a flow of airblast established by the opening of blast valve. The air-blast cools the arc and sweeps away the arcing products to the atmosphere. This rapidly increases the dielectric strength of the medium between contacts and prevents from reestablishing the arc. Consequently, the arc is extinguished and flow of current is interrupted.

\section{Disadvantages}

The use of air as the arc quenching medium offers the following disadvantages:

1. The air has relatively inferior arc extinguishing properties.

2. The air-blast circuit breakers are very sensitive to the variations in the rate of rise of restriking voltage.

3. Considerable maintenance is required for the compressor plant which supplies the air-blast.

The air blast circuit breakers are finding wide applications in high voltage installations. Majority of the circuit breakers for high voltage installations. Majority of the circuit breakers for voltages beyond $110 \mathrm{kV}$ are of this type.

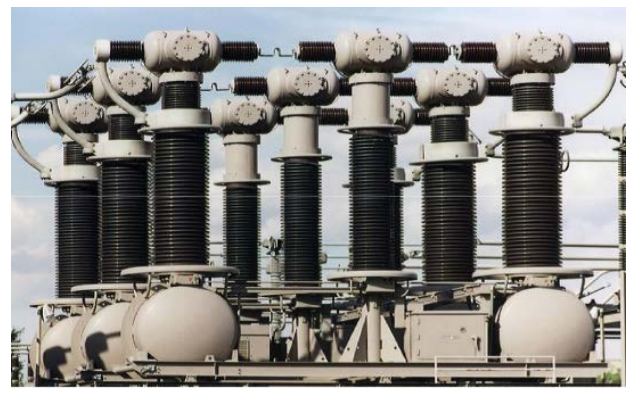

Figure 1: Air Insulated Circuit Breaker 


\section{B. Gas Circuit Breaker}

Sulphur Hexafluoride (SF6) Circuit Breakers:

In such circuit breakers, Sulphur hexafluoride (SF6) gas is used as the arc quenching medium. TheSF6 is an electronegative gas and has a strong tendency to absorb free electrons. The contacts of the breaker are opened in a high pressure flow of SF6 gas and an arc is struck between them.

\section{Advantages}

Due to the superior arc quenching properties of SF6 gas, the SF6 circuit breakers have many advantages over oil or air circuit breakers.

\section{Some of them are listed below}

1. Due to the superior arc quenching property of SF6, such circuit breakers have very Short arcing time.

2. Since the dielectric strength of SF6 gas is 2 to 3 times that of air, such breakers can interrupt much larger currents.

3. The SF6 circuit breaker gives noiselss operation due to its closed gas circuit and no exhaust to atmosphere unlike the air blast circuit breaker.

4. The closed gas enclosure keeps the interior dry so that there is no moisture

5. The closed gas enclosure keeps the interior dry so that there is no moisture problem.

6. There is no risk of fire in such breakers because SF6 gas is non-inflammable.

7. There are no carbon deposits so that tracking and insulation problems are eliminated.

8. The SF6breakers have low maintenance cost, light foundation requirements and minimum auxiliary equipment.

9. Since SF6breakers are totally enclosed and sealed from atmosphere, they are particularly suitable where explosion hazard exists e.g., coal mines.

\section{Disadvantages}

1. SF6 breakers are costly due to the high cost of SF6

2. Since SF6 gas has to be reconditioned after every operation of the breaker, additional equipment is required for this purpose.

\section{Electromechanical Relays}

Electric power utilities in Nigeria have traditionally used electromechanical (EMR) distance relays for the protection of transmission lines in the past, and many such relays are still in use in power systems today. These relays worked based on creating a mechanical force to operate the relay contacts in response to a fault situation. The mechanical force is established by the flow of a current that reflected the fault current through windings mounted in magnetic cores. Due to the nature of its principle of operation, electromechanical relays are relatively heavier and bulkier than relays constructed with other technologies. Its applications has become inadequate, insufficient and to a large extent, outdated in operations to meet the severe challenges imposed on modern twenty-first (21st) Century protective schemes. The size and complexity of modern power transmission and distribution systems, as well as the recent advances in digital computing, and protection relays technologies have made the manual operation of the relay settings impracticable. These relays have the following limitations:

1. Low speed of operation, single function Characteristic curve, and component failure leading to relay failure.

2. Excessive power consumption, Imposes high burden on instrument transformers,

3. No fault data available except phase indication.

Electromechanical relay is a hard wired relay. Its wiring is fixed, and the setting is manually performed. A calibrated adjustment called the time dial sets the spacing between the moving and stationary contacts, this varies the operating time of the relay from fast to slow.

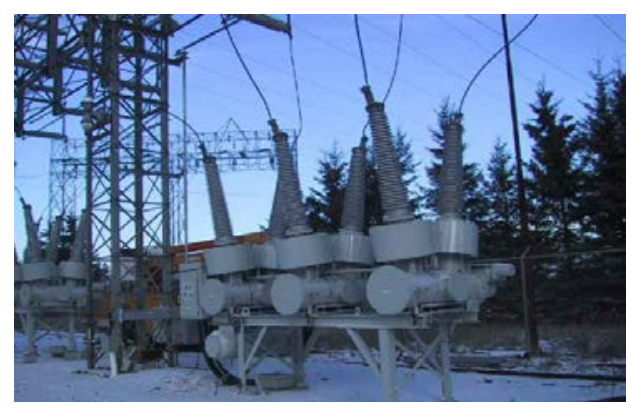

Figure 2: SF6 Circuit Breaker

\section{Numerical Relay}

Numerical relays are more flexible in configuring the relay settings, having better reliability, multifunctional capability, multiple relay setting characteristics, digital communication capabilities. Numerical relays are modular based so it can be easily serviced/ replaced, Numerical relays have very less burden, having better sensitivity/ selectivity, High speed operation (set/ reset), comprises auto resetting and self diagnostics features. Consumes very less space in comparison with electromechanical relays.

\section{System Evaluation}

\section{A. Substation Reference Drawing}

- For breaker replacement, we need to refer the following drawings.

- Single line diagram.

- Three line diagram.

1. Demo Elementary diagram.

2. Install Elementary diagram.

3. Demo Wiring diagram.

4. Install Wiring drawing.

5. Demo cable schedule.

6. Install cable schedule.

The above mentioned elementary/wiring diagram contains the below schematics and wiring drawings

1. AC Distribution.

2. DC Distribution.

3. Relay schematics.

4. Alarm Schematics.

5. Breaker control circuit.

(Breaker close/block circuit, TC-1 and TC-2 tripping circuit) CT \& PT circuit. 


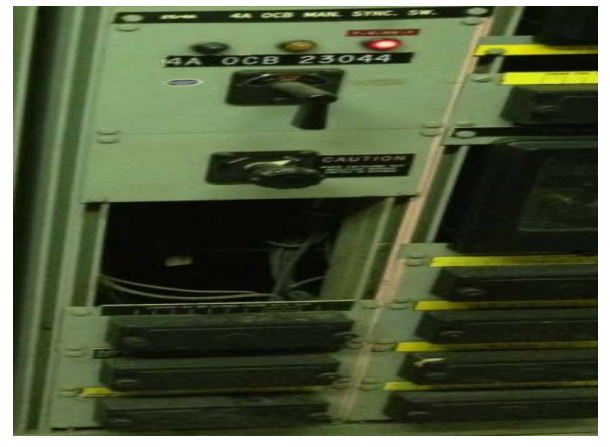

Figure 3(a)

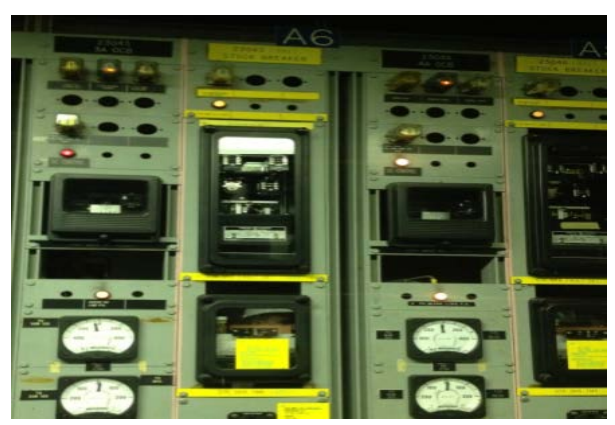

Figure 3(a) \& (b): Substation Site Walk-Down and Site Photos

\section{B. Preliminary Steps}

- Identifying the physical location of circuit breaker to be replaced with a new circuit breaker at the substation using single/three line diagram.

1. Analyze the existing breaker schematics which contains breaker close, tc- 1 , tc- 2 and all the associated tripping, status, alarm contacts which need to demoed and highlight the scope/field of replacement/deletion with green cloud with hatch. Previous phase install clouds and rev circles should be removed.

2. Repeat the same procedure for the electromechanical relays which needs to replaced with numerical relays. All electromechanical relay and associated contact distribution need to be demoed should be highlighted. The scope/field of replacement with green cloud with hatch.

3. By completing above two steps we will have the Demo elementary drawings in hand.

4. New approved breaker vendor drawing/Numerical relay contacts need to analyzed and the respective contact development can be made by using demo elementary drawing as input by deleting the content inside the green cloud and hatch with a new replacement/deletion of contacts/interlock logics and emphasizing the same with a red cloud which has undergone changes with the respective revision circle and revision details.

5. From the above steps from 1 to 4 we are ready with demo/install elementary schematics. These drawings will become guide line to make the changes in the demo/install wiring diagrams.

6. Need to gather all the required wiring drawings by referring to the front view diagrams of panel and the demo/elementary schematics. At any point we need to select appropriate wiring diagrams from the previous phase wiring diagrams install folder only otherwise drawing will be available in the read only folder. These wiring diagrams should be batch printed to get the hard copy which will be useful to carry out initial demo wiring markup.

7. To start with the demo wiring markup it requires to refer demo elementary drawings. Each and every markup from the demo elementary drawing need to be traced out point to point in panel wiring diagram and should also need to trace out remote end cables which are distributed to other panels through inter panel wiring, which needs to be demoed. Again for the demo we need to follow the same standard as demo elementary, green cloud with hatch. Previous phase install clouds and rev circles should be removed.

8. By completing the above step we are ready with demo wiring diagram. This will be the input to start the install wiring diagram. Demo wiring diagram should be cleaned up with respect to demo cloud and hatch and should be made ready for install markups. Demo wiring diagram should be batch printed to get hard copy of the same to start with install markups.

9. To start with install wiring need to refer install elementary drawings. Install elementary drawing will be the guideline to rebuild the protection and control logic for the new breaker by replacing the new set of breaker control circuit/contacts, numerical relay contacts and point to point connection without disturbing the unaffected part of the existing circuits. At any point we need to have only the respective cables in the install wiring as per install elementary schematics, all unnecessary cables should be removed. Like install elementary we need to follow the same standard of markup for the install wiring diagram with a red cloud, revision circle and revision details in the template.

10. After completing the above step we are ready with install wiring for the new breaker/numerical relay. Once demo/install wiring is completed, it requires to do yellow line check against Demo/Install elementary to make sure all the wiring is done as per elementariness.

11. Demo cable schedule can be completed by referring demo wiring diagram. Again for the demo we need to follow the same standard as demo elementary/wiring, green cloud with hatch. Previous phase install clouds and revision circles should be removed. This will become the input for install cable schedule markup.

12. For install cable schedule need to refer install wiring diagram. Demo cable schedule should be taken and need to clean up the diagram by deleting the demo revision cloud, hatch and the content inside it. New cables will be placed with latest cable number assigned to it and cables are grouped in to respective belonging group and same will be highlighted with red cloud.

13. With the above steps it completes existing breaker / electromechanical relay replacement with the new one. 


\section{Circuits Design}

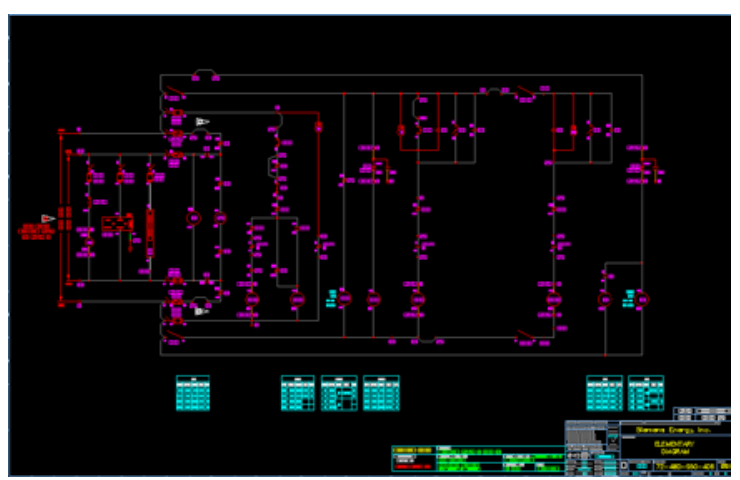

Figure 4: Vendor-Breaker Control Circuit

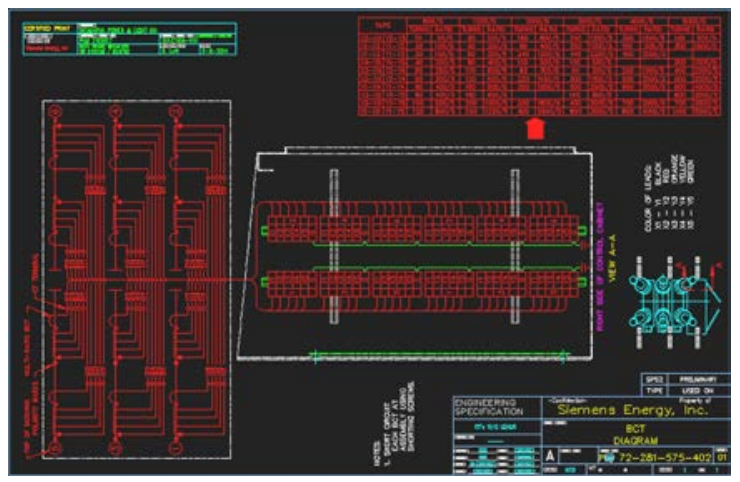

Figure 5: Vendor-CT Circuit

\section{Installation}

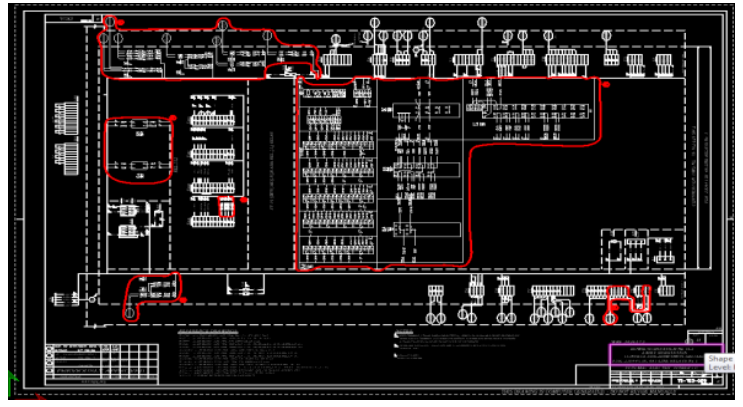

Figure 6: Install Wiring Diagram

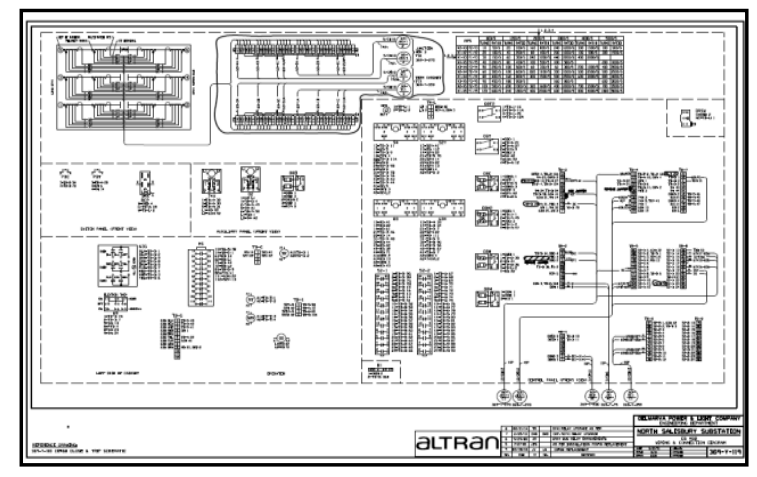

Figure 7: Breaker Control Wiring Diagram

\section{RELAYS ApPLICATIONS}

A typical SF6 circuit breaker consists of interrupter units each capable of dealing with currents upto $60 \mathrm{kA}$ and voltages in the range of $50-80 \mathrm{kV}$. A number of units are connected in series according to the system voltage. SF6circuit breakers have been developed for voltages $115 \mathrm{kV}$ to $230 \mathrm{kV}$, power ratings 10 MVA to 20 MVA and interrupting time less than 3 cycles.

Table 1: Comparison of Electro Mechanical and Numerical Relay

\begin{tabular}{|l|l|}
\hline \multicolumn{1}{|c|}{ Electromechanical } & \multicolumn{1}{|c|}{ Numerical } \\
\hline $\begin{array}{l}\text { Have fixed wiring and the setting is } \\
\text { manual }\end{array}$ & $\begin{array}{l}\text { Numerical relays are based on the } \\
\text { use of microprocessors }\end{array}$ \\
\hline $\begin{array}{l}\text { The values can be easily set. No } \\
\text { special programming device is } \\
\text { required }\end{array}$ & $\begin{array}{l}\text { Programmable relays where the } \\
\text { characteristics and behavior can be } \\
\text { programmed }\end{array}$ \\
\hline Multi functioning is not possible. & $\begin{array}{l}\text { Most numerical relays are also } \\
\text { multifunctional }\end{array}$ \\
\hline
\end{tabular}

\section{REFERENCES}

[1] National Electrical Code, NFPA 70, 2011.

[2] UL Standard for Safety for Molded-Case Circuit Breakers, Molded-Case Switches and Circuit-Breaker Enclosures, UL 489, 2009.

[3] IEEE Recommended Practice for Protection and Coordination of Industrial and Commercial Power Systems, IEEE Std 242-2001.

[4] UL Standard for Safety for Low-Voltage Fuses - Part 1: General Requirements, UL 248, 2011.

[5] Industrial Control Panels, UL 508A, 2005.

[6] Low-Voltage Switchgear and Control gear, IEC 60947-4-1-2000.

[7] UL Standard for Safety for Low-Voltage Fuses - Part 16: Test Limiters: UL 248, 2000.

[8] Semiconductor Fuse Application Guide, Mersen USA, 2002.

[9] IEEE Guide for Performing Arc-Flash Hazard Calculations, IEEE 1584-2002.

[10] Reducing Arc Flash Energies on Transformer Primaries, Tech Topics, Mersen USA, 2011.

[11] M.E. Valdes, C. Cline, S. Hansen and T. Papallo, "Selectivity analysis in low-voltage power distribution systems with fuses and circuit breakers", IEEE Trans. on Industry Applications, Vol. 46, No. 2, Pp.593-602, 2010.

[12] Handbook for Electrical Safety in the Workplace, NFPA 70E, 2012.

[13] J. Lewis, "Protective relaying: principles and applications", CRC Press, 2007.

[14] IEEE Recommend Practice for the Design of Reliable Industrial and Commercial Power Systems, IEEE Standard 493-2007.

[15] Achieving Higher Short Circuit Current Ratings for Industrial Control Panels, Tech Topics, Mersen USA, 2011.

[16] Enhancing Short Circuit Safety with Type 2 Coordination for Motor Starters, Tech Topics, Mersen USA, 2011. 